\title{
Electromagnetic fields and Green's functions in elliptical vacuum chambers
}

\author{
S. Persichelli* \\ Lawrence Berkeley National Laboratory, Berkeley, California 94720, USA \\ N. Biancacci \\ CERN, 1217 Meyrin, Geneva, Switzerland \\ M. Migliorati \\ CERN, 1217 Meyrin, Geneva, Switzerland \\ and University of Rome La Sapienza, and INFN Sez. Romal, 00185 Rome, Italy \\ L. Palumbo \\ University of Rome La Sapienza, and INFN Sez. Romal, 00185 Rome, Italy \\ V. G. Vaccaro \\ University of Napoli Federico II, and INFN Sez. di Napoli, 80138 Napoli, Italy
}

(Received 27 July 2017; published 23 October 2017)

\begin{abstract}
In this paper, we discuss the electromagnetic interaction between a point charge travelling inside a waveguide of elliptical cross section, and the waveguide itself. By using a convenient expansion of the Mathieu functions, useful in particular for treating a variety of problems in applied mathematics and physics with elliptic geometry, we first obtain the longitudinal electromagnetic field of a point charge (Green's function) in free space in terms of elliptical coordinates. This expression allows, then, to calculate the scattered field due to the boundary conditions in our geometry. By summing the contribution of the direct or primary field and the indirect field scattered by the boundary, after a careful choice of some expansion expressions, we derive a novel formula of the longitudinal electric field, in any transverse position of the elliptical cross section, generated by the charge moving along the longitudinal axis of the waveguide. The obtained expression is represented in a closed form, it can be differentiated and integrated, it can be used to fully describe the radiation process of a particle beam travelling inside a waveguide of elliptical cross section, and it is valid for any elliptic geometry. The equations are used to evaluate the coupling impedance due to indirect space charge in case of elliptical geometry. In addition, they are useful as preliminary studies for the determination of the coupling impedance in different cases involving elliptic vacuum chambers, as, for example, the effect of the finite conductivity of the beam pipe wall or the geometrical variation of the vacuum chamber due to elliptic step transitions existing in some accelerators.
\end{abstract}

DOI: 10.1103/PhysRevAccelBeams.20.101004

\section{INTRODUCTION}

A particle accelerator, from the electromagnetic point of view, can be thought as composed of several devices (for example rf cavities, magnets, beam diagnostics) connected by a vacuum chamber. A charged particle beam, travelling inside the accelerator and interacting with the surrounding environment, generates unwanted self-induced electromagnetic fields, which, under unfavorable conditions, perturb the beam motion, reduce the accelerator performances and,

\footnotetext{
*spersichelli@lbl.gov
}

Published by the American Physical Society under the terms of the Creative Commons Attribution 4.0 International license. Further distribution of this work must maintain attribution to the author(s) and the published article's title, journal citation, and DOI. in some cases, lead to instabilities. The collective effects of self-induced electromagnetic fields on the particle beam are generally studied by introducing the concepts of wakefield and coupling impedance [1]. It is then of paramount importance to have reliable tools that allow one to evaluate these self-induced fields. The intent of this paper is to derive an analytical expression able to describe the electromagnetic interaction between a charge travelling with arbitrary velocity inside a perfectly conducting beam pipe of elliptical cross section, and the pipe itself. These are called space charge fields. The analysis of this problem is primarily of interest for proton or ion machines, where space charge is important, and where it is not unusual to deal with elliptical geometries of the beam vacuum chamber [2-4]. In electron machines these effects are usually negligible. However, the expressions that we derive can also be of interest for them since the study can be 
extended to include the effects of the beam pipe finite conductivity [5,6], and to serve as a Green function for the problem of the impedance due to the geometrical variation of the vacuum chamber in an elliptic step transitions. In order to evaluate these electromagnetic fields, we introduce a set of special functions, useful for studying problems in applied mathematics and physics with elliptic geometries, called Mathieu functions. Mathieu functions were introduced in the literature in 1868 [7], when the vibrational modes of a membrane with an elliptic boundary were derived for the first time. In this paper we consider, as fundamental reference works on Mathieu functions, the books by McLachlan [8]. The computation of Mathieu functions is far from trivial [9], making the analysis of these functions more difficult than for Bessel functions. Nevertheless, an available source of software for the computation of Mathieu functions, that we have used for the field computation presented in this paper, is described in [10].

The paper is organized as follows: in Sec. II we present a short theoretical introduction on elliptical coordinates and Mathieu functions, the wave equation in elliptical coordinates generated by a charged particle beam and its general solutions, together with some notations and normalizations adopted in this paper. In Sec. III we derive the primary field, that is the field directly generated by a point charge in the free space, and the scattered field, that is the field reflected by the metallic walls and resulting from the boundary conditions of the vacuum chamber. Since these fields, which we manage to express with separate variables of the elliptical coordinates, are given in terms of infinite summations, an optimization of the numerical convergence of these series has also been performed. In Sec. IV we derive the expression, for an arbitrary transverse position, of the longitudinal electric field produced by a point charge travelling on the axis of the elliptical beam pipe, and we also show some numerical computation examples applied to two accelerators of the CERN complex, the Proton Synchrotron and the Super Proton Synchrotron. In Secs. V and VI we apply the expression of the scattered field to obtain the longitudinal and transverse quadrupolar indirect space charge coupling impedance for any elliptic geometry. Finally, Sec. VII is dedicated to concluding remarks.

\section{WAVE EQUATION IN ELLIPTICAL COORDINATES}

Unlike in the classical approach of propagation of electromagnetic fields in elliptical waveguides [11,12], in this paper we take, as field source, a charged particle beam moving with velocity $v=\beta c$, with $c$ the speed of light, along the longitudinal axis (z-axis) of a perfectly conducting elliptical vacuum chamber. Under this condition, if we consider a transverse magnetic (TM) mode propagating along $\mathrm{z}$, the wave equation is given by [13]

$$
\nabla_{t}^{2} E_{z}^{0}+\left(k_{0}^{2}-k_{z}^{2}\right) E_{z}^{0}=0
$$

where $\nabla_{t}^{2}$ is the transverse bi-dimensional Laplacian, $E_{z}^{0}$ the longitudinal component of the electric field, $k_{0}$ the wave number in free space equal to $\omega / c$, and $k_{z}$ the propagation constant, imposed by the beam velocity and equal to $\omega / c \beta$. The above equation is valid for a waveguide with arbitrary cross-section. The longitudinal dependence of the field with $z$ is of the kind $e^{-j k_{z} z}$. In the following we omit this propagation term to simplify notation.

The main difference of Eq. (1) from the common approach of mode propagation in an elliptic waveguide $[11,12]$, is that here $k_{z}$ is not an unknown of our problem. The transverse waves generated by the beam are in cut off, namely, by defining $k_{t}^{2}=k_{0}^{2}-k_{z}^{2}$, we observe that this difference is negative, and therefore $k_{t}=\sqrt{k_{0}^{2}-k_{z}^{2}}$ is an imaginary quantity.

If we consider an elliptical cross-section, it is convenient to describe the geometry using confocal elliptical coordinates. In this coordinate system, shown in Fig. 1, we define the angular coordinate $\varphi$, that describes a set of hyperbolas having the same foci, and the radial coordinate $\mu$ which gives a set of confocal ellipses. The elliptic variable $\varphi$ has a domain $0 \leq \varphi \leq 2 \pi$, and plays a similar role of an angular variable in polar coordinates. The variable $\mu$, in the domain $0 \leq \mu<\infty$, behaves like a radial variable.

The relation between elliptical and Cartesian coordinates is given by [14]

$$
\begin{cases}x=F & \cosh \mu \cos \varphi \\ y=F & \sinh \mu \sin \varphi\end{cases}
$$

where $F$ is the focal distance of the ellipse, related to the semimajor and semiminor axes $a$ and $b$ by

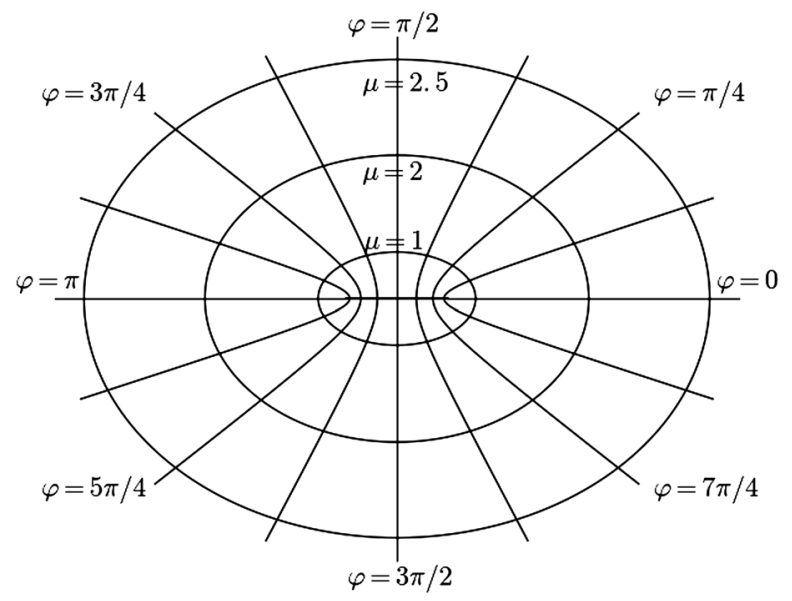

FIG. 1. Elliptic coordinates. The $\varphi$ coordinate describes confocal hyperbolas that are symmetrical about the $\mathrm{x}$-axis. The $\mu$ coordinate describes confocal ellipses centered on the origin of the coordinate system. 


$$
F=\sqrt{a^{2}-b^{2}} .
$$

The line joining the foci corresponds to the case $\mu=0$ and $0 \leq \varphi \leq \pi$, and the origin of our coordinate system is located in $\mu=0, \varphi=\pi / 2$. In our notation, the elliptical boundary of the beam pipe can be written in terms of the eccentricity $e$ of the ellipse by

$$
e=\frac{\sqrt{a^{2}-b^{2}}}{a}=\frac{1}{\cosh \mu_{0}} .
$$

The polar coordinates can be considered a special case of the elliptic coordinates in the limit $F \rightarrow 0$, when the foci collapse into the origin.

The bi-dimensional Laplacian of Eq. (1) in this coordinate system can be written as [15]:

$$
\nabla_{t}^{2}=\frac{2}{F^{2}(\cosh 2 \mu-\cos 2 \varphi)}\left(\frac{\partial^{2}}{\partial \mu^{2}}+\frac{\partial^{2}}{\partial \varphi^{2}}\right) .
$$

If we substitute the above Laplacian definition in Eq. (1), we obtain the wave equation in elliptical coordinates:

$\frac{2}{F^{2}(\cosh 2 \mu-\cos 2 \varphi)}\left(\frac{\partial^{2}}{\partial \mu^{2}}+\frac{\partial^{2}}{\partial \varphi^{2}}\right) E_{z}^{0}+k_{t}^{2} E_{z}^{0}=0$,

where $k_{t}^{2}$ is negative. It is worth noting that Eq. (6) cannot be written as a standard equation of propagation of electromagnetic modes in an elliptical waveguide, as (18.50) in [8]. However, if we define

$$
q=-\frac{k_{t}^{2} F^{2}}{4}>0
$$

a formal solution of Eq. (6) can still be obtained by using the same method of variable separation. This method, in elliptical coordinates, gives two ordinary differential equations, involving a separation constant $a$ :

$$
\begin{gathered}
\frac{d^{2} V}{d \varphi^{2}}+(a+2 q \cos 2 \varphi) V=0 \\
\frac{d^{2} U}{d \mu^{2}}-(a+2 q \cosh 2 \mu) U=0 .
\end{gathered}
$$

Equations (8) and (9) are known, respectively, as ordinary and modified Mathieu equations of the first kind of integral order with changed sign in $q$ (see, e.g., 2.18(1), and 2.31(1) in [8]). In the limit $F \rightarrow 0$, when the foci of the elliptic coordinates collapse into the origin, the angular and radial Mathieu equations become harmonic and Bessel equations, respectively. It exists a countably infinite set of characteristic values $a(q)$ which yield even periodic solutions of Eq. (8). These solutions can be expressed with four categories of periodic ordinary Mathieu functions as follows [8]:

$$
\begin{aligned}
c e_{2 n}(\varphi,-q) & =(-1)^{n} \sum_{r=0}^{\infty}(-1)^{r} A_{2 r}^{(2 n)} \cos (2 r \varphi) \\
c e_{2 n+1}(\varphi,-q) & =(-1)^{n} \sum_{r=0}^{\infty}(-1)^{r} A_{2 r+1}^{(2 n+1)} \cos [(2 r+1) \varphi] \\
\operatorname{se}_{2 n+1}(\varphi,-q) & =(-1)^{n} \sum_{r=0}^{\infty}(-1)^{r} B_{2 r+1}^{(2 n+1)} \sin [(2 r+1) \varphi] \\
\operatorname{se}_{2 n+2}(\varphi,-q) & =(-1)^{n} \sum_{r=0}^{\infty}(-1)^{r} B_{2 r+2}^{(2 n+2)} \sin [(2 r+2) \varphi],
\end{aligned}
$$

with $n$ defining the order of the Mathieu functions. The expansion coefficients in the above series, $A$ and $B$, which are functions of $q$, are defined in such a way that the angular functions $c e_{n}$ and $s e_{n}$ are orthogonal [8]. Furthermore, the Mathieu functions are normalized according to the following equation:

$$
\int_{0}^{2 \pi} c e_{n}(z,-q) c e_{p}(z,-q) d z=\left\{\begin{array}{ll}
0 & n \neq p \\
\pi & n=p
\end{array} .\right.
$$

A similar expression is valid for $s e_{n}$.

The expansion coefficients in Eq. (10) can be obtained by substituting the above series in the differential equation (8), leading to an infinite homogeneous system of linear equations. For example, for the coefficients $A_{2 m}^{(2 n)}$, it is possible to obtain the three-term recursion relations

$$
\begin{aligned}
a A_{0}^{(2 n)}-q A_{2}^{(2 n)} & =0 \\
{\left[a-(2 m)^{2}\right] A_{2 m}^{(2 n)}-q\left(A_{2 m-2}^{(2 n)}+A_{2 m+2}^{(2 n)}\right) } & =0 \quad(m \geq 2) .
\end{aligned}
$$

For a fixed $q$, this becomes a tri-diagonal matrix equation representing an eigenvalue problem in $A$ with the coefficients $A_{2 m}^{(2 n)}$, which are the eigenvector components. Since the terms $A_{2 m}^{(2 n)}$ are negligibly small as $m$ becomes very large, the matrix can be truncated and a finite eigenvalue problem can then be solved. Similar expressions can also be obtained for the other coefficients in Eq. (10).

Solutions of Eq. (9) can be obtained from Eq. (8) by setting the change of variable $\varphi=j \mu$, with $j$ the imaginary unit. The solutions are called radial modified Mathieu functions of the first kind, and can be defined as follows [8]: 


$$
\begin{aligned}
C e_{2 n}(\mu,-q) & =(-1)^{n} \sum_{r=0}^{\infty}(-1)^{r} A_{2 r}^{(2 n)} \cosh (2 r \mu) \\
C e_{2 n+1}(\mu,-q) & =(-1)^{n} \sum_{r=0}^{\infty}(-1)^{r} A_{2 r+1}^{(2 n+1)} \cosh [(2 r+1) \mu] \\
S e_{2 n+1}(\mu,-q) & =(-1)^{n} \sum_{r=0}^{\infty}(-1)^{r} B_{2 r+1}^{(2 n+1)} \sinh [(2 r+1) \mu] \\
S e_{2 n+2}(\mu,-q) & =(-1)^{n} \sum_{r=0}^{\infty}(-1)^{r} B_{2 r+2}^{(2 n+2)} \sinh [(2 r+2) \mu] .
\end{aligned}
$$

Finally, the solution of Eq. (6) is given by a combination of the above Mathieu functions of even or odd order [11]:

$$
E_{z}^{0}(\mu, \varphi)=\sum_{r=0}^{\infty}\left\{\begin{array}{c}
a_{r} c e_{r}(\varphi,-q) C e_{r}(\mu,-q) \\
b_{r} s e_{r+1}(\varphi,-q) S e_{r+1}(\mu,-q)
\end{array}\right\} .
$$

The solution of the wave equation, in order to satisfy the boundary conditions, has to be null on the contour of the ellipse: $E_{z}^{0}\left(\mu_{0}, \varphi\right)=0$, with $\mu_{0}$ defining the elliptical boundary given by Eq. (4).

In addition to the above equations, it is convenient to introduce here also the radial modified Mathieu functions of second kind $\mathrm{Fek}_{2 n}(\mu,-q)$ [8]. These functions play a role in elliptic coordinates similar to the modified Bessel functions of the second kind $K_{n}$ for cylindrical coordinates. Indeed, as the pair of Bessel functions $I_{0}$ and $K_{0}$, with $I_{0}$ the zero order modified Bessel function of the first kind, are used to express the electromagnetic field generated by a charged particle in cylindrical symmetry [16], the pair $\mathrm{Ce}_{2 n}$ and $\mathrm{Fek}_{2 n}$ will be used here for our case in elliptic coordinates. The functions $\operatorname{Fek}_{2 n}(\mu,-q)$ are defined in terms of series of the Bessel functions $K_{n}$ as in 8.20 of [8]:

$$
\begin{aligned}
F e k_{2 n}(\mu,-q)=( & -1)^{n} \frac{c e_{2 n}\left(\frac{\pi}{2}, q\right)}{\pi A_{0}^{(2 n)}} \sum_{r=0}^{\infty} A_{2 r}^{(2 n)} K_{2 r}(2 \sqrt{q} \sinh \mu) \\
F e k_{2 n+1}(\mu,-q)= & (-1)^{n} \frac{\operatorname{se} 2 n+1\left(\frac{\pi}{2}, q\right)}{\pi \sqrt{q} B_{1}^{(2 n+1)}} \operatorname{coth} \mu \\
& \times \sum_{r=0}^{\infty}(2 r+1) B_{2 r+1}^{(2 n+1)} K_{2 r+1}(2 \sqrt{q} \sinh \mu)
\end{aligned}
$$

with the condition $|\sinh \mu|>1$, necessary for absolute and uniform convergence of the expansions [8]. Similar expressions with different coefficients can also be written by using $\cosh \mu$ as argument of the Bessel functions instead of $\sinh \mu$. In addition to the above expressions, it is possible to demonstrate that the functions $F e k_{2 n}(\mu,-q)$ can be also expressed in terms of product series of Bessel functions $I_{n}(x) K_{n}(x)$. For example, we can write [8]:

$$
\operatorname{Fek}_{2 n}(\mu,-q)=\frac{p_{2 n}^{\prime}}{\pi A_{0}^{(2 n)}} \sum_{r=0}^{\infty} A_{2 r}^{(2 n)} I_{r}\left(\nu_{1}\right) K_{r}\left(\nu_{2}\right)
$$

with

$$
p_{2 n}^{\prime}=(-1)^{n} \frac{c e_{2 n}(0, q) c e_{2 n}\left(\frac{\pi}{2}, q\right)}{A_{0}^{(2 n)}},
$$

and $\nu_{1}=\sqrt{q} e^{-\mu}$ and $\nu_{2}=\sqrt{q} e^{\mu}$. The product series (17) converges more rapidly than those with arguments $2 \sqrt{q} \sinh \mu, 2 \sqrt{q} \cosh \mu$, and it converges uniformly in any finite region of the $\mu$-plane [8]. By virtue of this, the product series is preferable for calculating the values of the functions $\mathrm{Fek}_{2 n}(\mu,-q)$.

\section{THE RADIATION PROBLEM IN AN ELLIPTICAL WAVEGUIDE}

In this section, we derive an analytical expression of the longitudinal electric field generated by a Dirac $\delta$-function beam distribution with charge $Q$ travelling in free space. Of course, this is a well known and solved problem, but, in order to match the elliptical geometry that we are considering for the beam pipe, we want to express the field in elliptical coordinates and with separate functions of the variables $\varphi$ and $\mu$. Let us first consider the following wave equation of the electric field $E_{z}^{0}$ for a TM mode generated by the charge moving along the axis of the pipe cross-section:

$$
\nabla_{t}^{2} E_{z}^{0}+k_{t}^{2} E_{z}^{0}=-G \delta(x) \delta(y),
$$

where $\delta$ is the Dirac delta function and $G$ is a constant that depends on the beam parameters and can be calculated as [13]

$$
G=j Z_{0} \frac{Q k_{0}}{2 \pi \beta^{2} \gamma^{2}},
$$

where $Z_{0}$ is the characteristic impedance in free space and $\gamma$ is the Lorentz factor.

As we have already discussed at the beginning of Sec. II, the transverse wave number $k_{t}$ is an imaginary quantity and it can be written here as

$$
k_{t}=j \frac{k_{0}}{\beta \gamma} .
$$

The electric field $E_{z}^{0}$ can be represented as the superposition of a field generated by the particle beam in free space and a field that is scattered by the boundary of the waveguide and that acts back on the beam itself. Therefore, 
we can divide the solution of the wave equation into two scalar solutions:

$$
E_{z}^{0}=E_{z}^{s}+E_{z}^{i}
$$

The first scalar solution $E_{z}^{s}$ represents the direct (or primary) component of the field. It has to satisfy the inhomogeneous wave equation in free space

$$
\nabla_{t}^{2} E_{z}^{s}-\frac{k_{0}^{2}}{\beta^{2} \gamma^{2}} E_{z}^{s}=-G \delta(x) \delta(y),
$$

and it has to satisfy the conditions of radiation at infinity. At the origin of the elliptical coordinates the direct field diverges.

The second scalar solution in Eq. (22), $E_{z}^{i}$, is called the indirect field, and it is the field scattered from the boundary, which, in our case, is elliptic. The indirect field has to satisfy the homogeneous wave equation

$$
\nabla_{t}^{2} E_{z}^{i}-\frac{k_{0}^{2}}{\beta^{2} \gamma^{2}} E_{z}^{i}=0
$$

and it has a finite value at the origin of the elliptical coordinates.

In the following subsections, we are going to calculate separately the two solutions of the wave equations (23) and (24) in elliptical geometry, using the condition that the sum of the direct and indirect field must satisfy the boundary conditions on the contour of the beam pipe.

\section{A. Direct field or Green's function in free space}

An expression of the direct field in free space generated by a Dirac $\delta$ distribution in cylindrical geometry, that is a solution of Eq. (23), is given by [13]

$$
E_{z}^{s}=G K_{0}\left(\frac{k_{0} r}{\beta \gamma}\right),
$$

where $r$ is the radial coordinate and $K_{0}$ is the zero order modified Bessel function of second kind. This expression allows us to calculate the Green's function at any position in free space. We want to derive an equivalent expression in elliptical coordinates. To do that, we first substitute the radial polar coordinate $r$ with Eq. (2), and then obtain the argument of the Bessel function:

$$
\begin{aligned}
K_{0} & \left(\frac{k_{0} F}{\beta \gamma} \sqrt{\sinh ^{2} \mu+\cos ^{2} \varphi}\right) \\
& =K_{0}\left(\frac{k_{0} F}{2 \beta \gamma} \sqrt{e^{2 \mu}+e^{-2 \mu}-2 \cos (2 \varphi+\pi)}\right) .
\end{aligned}
$$

Then, by using the Gegenbauer's addition theorem [17], it is possible to expand the modified Bessel function $K_{0}$ as [18]:

$$
\begin{array}{r}
K_{0}\left(\frac{k_{0} F}{2 \beta \gamma} \sqrt{e^{2 \mu}+e^{-2 \mu}-2 \cos (2 \varphi+\pi)}\right) \\
=\sum_{n=0}^{\infty}(-1)^{n} \epsilon_{n} I_{n}\left(z_{1}\right) K_{n}\left(z_{2}\right) \cos (2 n \varphi),
\end{array}
$$

where $\epsilon_{n}=\frac{2}{1+\delta_{n, 0}}$, with $\delta_{n, 0}$ the Kronecker delta and

$$
z_{1}=\frac{k_{0} F}{2 \beta \gamma} e^{-\mu} ; \quad z_{2}=\frac{k_{0} F}{2 \beta \gamma} e^{\mu} .
$$

It is worth noting that, by the use of Eq. (27), we have now provided an expansion of $E_{z}^{s}$ in separate functions of $\mu$ and $\varphi$. However, the equation needs to be further manipulated in order to express the electric field in terms of Mathieu functions, which represent a natural expression of the fields in elliptical geometry.

Following the procedure described in [19], applying the method of Ritz-Galerkin [20] and the orthogonality properties of Mathieu's functions, it is possible to expand the cosine function in Eq. (27) as

$$
\begin{aligned}
\epsilon_{n} \cos (2 n \varphi) & =2(-1)^{n} \sum_{l=0}^{\infty}(-1)^{l} A_{2 n}^{(2 l)} c e_{2 l}(\varphi,-q) \\
& =2(-1)^{n} \sum_{l=0}^{\infty} A_{2 n}^{(2 l)} c e_{2 l}\left(\frac{\pi}{2}-\varphi, q\right),
\end{aligned}
$$

with an arbitrary value of $q$. By changing $q$, both the coefficients $A_{2 n}^{(2 l)}$ and the periodic ordinary Mathieu functions change accordingly, and the equation remains satisfied. If we now introduce Eq. (29) in Eq. (27), the electric field is given by:

$E_{z}^{s}=2 G \sum_{l=0}^{\infty} c e_{2 l}\left(\frac{\pi}{2}-\varphi, q\right) \sum_{n=0}^{\infty} A_{2 n}^{(2 l)} I_{n}\left(z_{1}\right) K_{n}\left(z_{2}\right)$.

Equation (30) is an alternative representation of Eq. (25), and, written in this form, $q$ is still arbitrary. If $q$ is defined as

$$
q=\left(\frac{k_{0} F}{2 \beta \gamma}\right)^{2}
$$

the inner sum in Eq. (30) is proportional to the radial modified Mathieu functions of second kind $\mathrm{Fek}_{2 l}(\mu,-q)$ of Eq. (17). We can therefore express the Green's function in compact form as:

$E_{z}^{s}=2 \pi G \sum_{l=0}^{\infty} \frac{A_{0}^{(2 l)}}{p_{2 l}^{\prime}} c e_{2 l}\left(\frac{\pi}{2}-\varphi, q\right) F e k_{2 l}(\mu,-q)$.

Equation (32) is our result for the direct electric field as solution of Eq. (23). It is expressed in terms of a product of Mathieu functions with separate elliptical coordinates $\varphi$ 


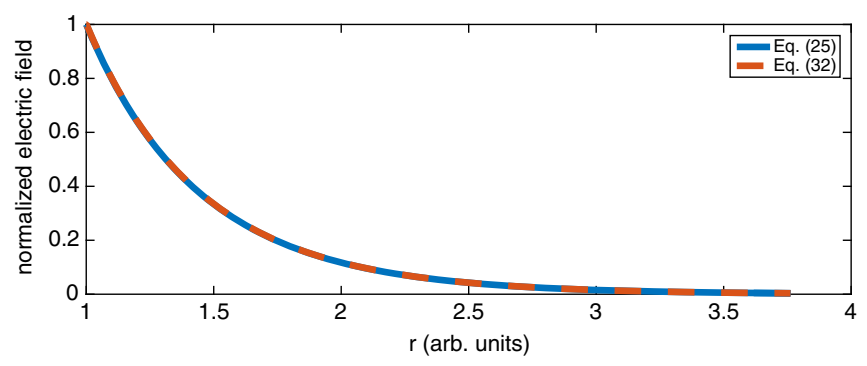

FIG. 2. Comparison between the Green's function in free space computed with Eq. (25) (blue line) and Eq. (32) (red dashed line) with 120 summation terms, as a function of the radial coordinate and $\varphi=0$, for a focal distance $F=1$.

and $\mu$. Since we have derived the expression directly from the Green's function in free space, it satisfies all the properties of the Green's function. Written in this form, it is possible to verify that it is rapidly convergent because the coefficients $A_{0}^{(2 l)}$ go rapidly to zero. Since the electric field must exhibit the same configuration independently from the coordinate system that has been adopted, we can compare Eq. (32), expanded in elliptical coordinates, with Eq. (25), written in terms of cylindrical coordinates.

Figures 2 and 3 show the numerical comparison between the two expressions of the electric field at $\varphi=0(r=x \geq F)$ and $\varphi=\pi / 2(r=y>0)$ as a function of the distance from the origin. The relations between $r$ ( $x$ or $y$ ) and $\mu$ are given by Eq. (2). For the numerical computation, we considered a focal distance $F=1$, but any value of $F$ can be used. In both cases, we summed 120 terms, for which the calculation is almost immediate on an ordinary desktop PC. These two examples show that the two curves agree very well.

\section{B. Indirect field (scattered field)}

The indirect field has to satisfy the homogeneous wave equation (24). Since we are dealing with elliptical geometry, in order to simplify the application of the boundary conditions, it is better to represent the component of the

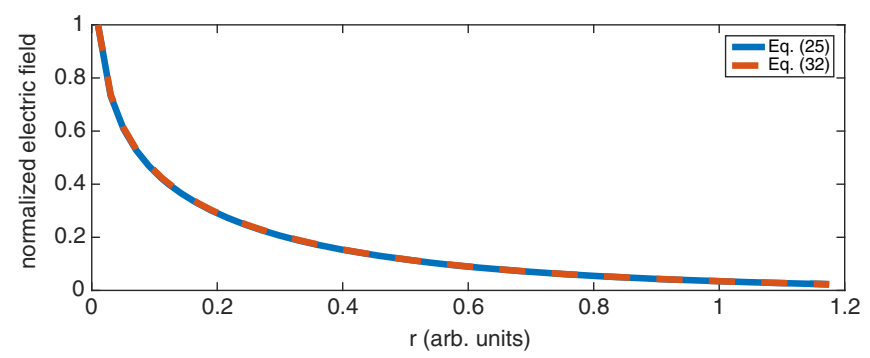

FIG. 3. Comparison between the Green's function in free space computed with Eq. (25) (blue line) and Eq. (32) (red dashed line) with 120 summation terms, as a function of the radial coordinate and $\varphi=\pi / 2$, for a focal distance $F=1$. indirect electric field $E_{z}^{i}$ directly as expansion of Mathieu radial and angular functions, as in Eq. (14), that is

$$
\begin{aligned}
E_{z}^{i} & =2 \pi G \sum_{l=0}^{\infty} M_{2 l}(-1)^{l} c e_{2 l}(\varphi,-q) C e_{2 l}(\mu,-q) \\
& =2 \pi G \sum_{l=0}^{\infty} M_{2 l} c e_{2 l}\left(\frac{\pi}{2}-\varphi, q\right) C e_{2 l}(\mu,-q),
\end{aligned}
$$

with $M_{2 l}$ the unknown amplitude of the field. The constant $2 \pi G$ and the term $(-1)^{l}$ have been introduced here to simplify the final expression of the total electric field. It is important to underline the similarity of Eq. (33) with Eq. (32). The total field $E_{z}^{0}$ of Eq. (22), representing the electric field produced by a charged beam in an elliptical vacuum chamber, is, therefore, given by the sum of the direct and indirect field:

$$
\begin{aligned}
E_{z}^{s}+E_{z}^{i}= & 2 \pi G \sum_{l=0}^{\infty} \frac{A_{0}^{(2 l)}}{p_{2 l}^{\prime}} c e_{2 l}\left(\frac{\pi}{2}-\varphi, q\right) F e k_{2 l}(\mu,-q) \\
& +2 \pi G \sum_{l=0}^{\infty} M_{2 l} c e_{2 l}\left(\frac{\pi}{2}-\varphi, q\right) C e_{2 l}(\mu,-q) .
\end{aligned}
$$

In order to satisfy the boundary conditions, we have to impose, in the above equation, a zero value electric field $E_{z}^{0}$ on the elliptic surface $\mu_{0}$, expressed by Eq. (4), for any value of $\varphi$. This gives a condition for the unknown amplitudes $M_{2 l}$, which must be such that:

$$
\begin{aligned}
2 \pi G & {\left[\sum_{l=0}^{\infty} \frac{A_{0}^{(2 l)}}{p_{2 l}^{\prime}} c e_{2 l}\left(\frac{\pi}{2}-\varphi, q\right) \text { Fek }_{2 l}\left(\mu_{0},-q\right)\right.} \\
+ & \left.\sum_{l=0}^{\infty} M_{2 l} c e_{2 l}\left(\frac{\pi}{2}-\varphi, q\right) C e_{2 l}\left(\mu_{0},-q\right)\right]=0 .
\end{aligned}
$$

The above relation has to be satisfied independently for each term of the summation. Therefore, the unknown amplitude coefficients of the indirect field can be calculated as:

$$
M_{2 l}=-\frac{A_{0}^{(2 l)}}{p_{2 l}^{\prime}} \frac{F e k_{2 l}\left(\mu_{0},-q\right)}{C e_{2 l}\left(\mu_{0},-q\right)} .
$$

By considering the previous condition on the amplitudes $M_{2 l}$, we can now fully describe the indirect (scattered) field in terms of a combination of periodic ordinary and radial modified Mathieu functions of first kind:

$$
\begin{aligned}
E_{z}^{i}= & -2 \pi G \sum_{l=0}^{\infty} \frac{A_{0}^{(2 l)}}{p_{2 l}^{\prime}} \frac{F e k_{2 l}\left(\mu_{0},-q\right)}{C e_{2 l}\left(\mu_{0},-q\right)} c e_{2 l}\left(\frac{\pi}{2}-\varphi, q\right) \\
& \times C e_{2 l}(\mu,-q) .
\end{aligned}
$$




\section{TOTAL FIELD IN ELLIPTICAL COORDINATES}

The total field $E_{z}^{0}$ excited by a Dirac $\delta$ beam distribution in a beam pipe of elliptic cross section is finally expressed in terms of product series of periodic ordinary Mathieu functions $\mathrm{Ce}_{2 l}$ and radial modified Mathieu functions $\mathrm{Fek}_{2 l}$ and $\mathrm{Ce}_{2 l}$. Combining Eqs. (32) and (37), the expansion of the total field is given by

$$
\begin{aligned}
E_{z}^{0}= & 2 \pi G \sum_{l=0}^{\infty} \frac{A_{0}^{(2 l)}}{p_{2 l}^{\prime}} c e_{2 l}\left(\frac{\pi}{2}-\varphi, q\right)\left[F e k_{2 l}(\mu,-q)\right. \\
& \left.-\frac{F e k_{2 l}\left(\mu_{0},-q\right)}{C e_{2 l}\left(\mu_{0},-q\right)} C e_{2 l}(\mu,-q)\right]
\end{aligned}
$$

that satisfies the boundary conditions on the contour of the ellipse representing the beam vacuum chamber. Equation (38) is our final result, expressed in a compact form, to calculate the longitudinal electric field, at any frequency and transverse position, produced by a point charge in an elliptic vacuum chamber of any dimension and aspect ratio. An equivalent expression could also be obtained by using an expansion of the eigenmodes in the elliptic waveguide. In Appendix A we show the procedure that should be used, and we explain the reasons why we did not follow that method.

It is important to observe that Eq. (38) is formally very similar to the well-known longitudinal electric field generated by a point charge travelling on the axis of a perfectly conducting beam pipe of radius $r_{0}$ [13]:

$$
E_{z}^{0}=G\left[K_{0}\left(\frac{k_{0} r}{\beta \gamma}\right)-\frac{K_{0}\left(\frac{k_{0} r_{0}}{\beta \gamma}\right)}{I_{0}\left(\frac{k_{0} r_{0}}{\beta \gamma}\right)} I_{0}\left(\frac{k_{0} r}{\beta \gamma}\right)\right],
$$

with the pair of modified Bessel functions of first and second order $I_{0}-K_{0}$ replacing the pair of modified Mathieu functions of first and second order $\mathrm{Ce}_{2 l}-\mathrm{Fek}_{2 l}$. The equation can therefore be used to fully describe and rapidly compute the longitudinal electric field produced by a charge moving along the $\mathrm{z}$-axis of a beam vacuum chamber having elliptical cross sections.

For completeness, we show in Appendix B that Eq. (39) can be derived from Eq. (38) in the limit of $q \rightarrow 0$ (or $k_{0} \rightarrow 0$ ). An alternative way of deriving the low frequency limit of Eq. (39) and Eq. (38), based on field decomposition in terms of eigenfunctions and eigenvalues of a $2 \mathrm{D}$ boundary problem, is discussed in [21].

Using an expression containing the radial modified Mathieu functions of second kind $F e k_{2 l}$ presents several advantages. The field is expressed in a compact analytical form that can be used as a first step to obtain more complicated fields in elliptic geometry, as, for example, including finite resistivity of the beam vacuum chamber. In addition, formal expressions for the derivative and the integral of the electric field can be easily written. Moreover, it is worth noting that the summation in Eq. (38) converges very rapidly.

A different expression of the electric field in an elliptical vacuum chamber, with and without losses, has already been derived in [22]. Nevertheless, a different approach considering a Gaussian beam distribution (not a point charge) has been used, leading to a more complicated formulation of the field.

In order to validate Eq. (38), we have compared our results with those obtained from the electromagnetic code CST Particle Studio [23]. With CST, we can define an elliptic vacuum chamber with perfectly conducting pipe, and compute the electromagnetic interaction between a Gaussian beam of a fixed charge and energy, travelling along the longitudinal axis of the pipe, and its surrounding environment. The CST frequency monitor allows to compute the electric or magnetic field along any user defined curve or inside a volume, at a given frequency. By the use this monitor, we computed the longitudinal electric field along an arbitrary ellipse inside the geometry. The field computed by CST is given by the sum of the direct and the indirect field, and therefore it is not possible to separate the two contributions. In addition to that, the use of CST for this type of computation is not very suited in case of nonrelativistic energies $(\beta<1)$, since the computation can result very time consuming and the low value of the resulting electric field can, in some cases, be altered by numerical noise.

In Fig. 4, a comparison between the field per unit of charge computed with Eq. (38) and by CST, at a frequency of $100 \mathrm{MHz}$ and $\mu=0.1$, is shown as a function of $\varphi$. In this example, we considered the CERN Proton Synchrotron (PS) machine, that is designed with an elliptic beam pipe [2] with $a=7.3 \mathrm{~cm}$ and $b=3.5 \mathrm{~cm}$, and $\beta=0.91$. The two results are in very good agreement, and the formula converges to the simulation result with 40 summation terms, taking just a few seconds of calculation. By testing

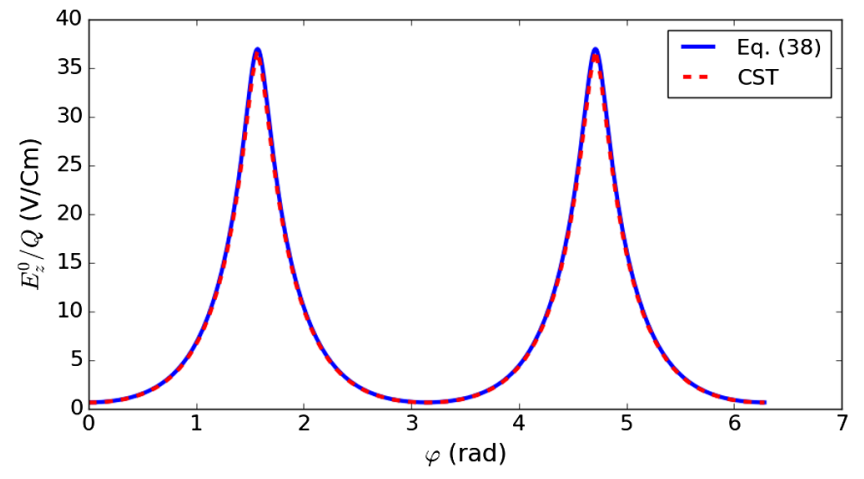

FIG. 4. Comparison between the longitudinal electric field per unit of charge computed with Eq. (38) (blue line) and by CST (red dashed line) with 40 summation terms, calculated in $\mu=0.1$ at $100 \mathrm{MHz}$ as a function of $\varphi$. 


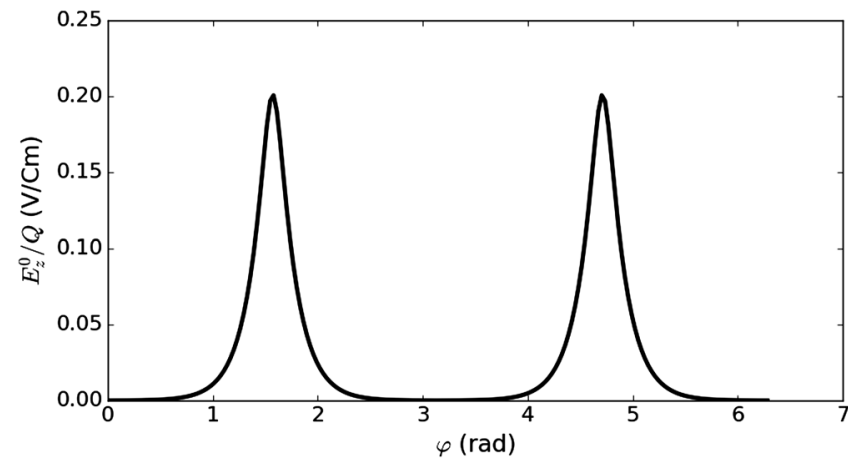

FIG. 5. Longitudinal electric field per unit of charge in the elliptic beam pipe of the SPS accelerator at the injection energy, calculated in $\mu_{0}=0.1$ at $100 \mathrm{MHz}$ as a function of $\varphi$.

different geometries and beam characteristics, we observed an excellent agreement between the analytical formula and the simulation results in a variety of cases.

In Fig. 5, an example of the total longitudinal electric field per unit of charge, calculated as a function of the coordinate $\varphi$ for $\mu=0.1$ at a frequency of $100 \mathrm{MHz}$, is shown for the CERN Super Proton Synchrotron (SPS). The SPS is designed with an elliptic beam pipe having $a=7.8 \mathrm{~cm}$ and $b=2.1 \mathrm{~cm} \mathrm{[24]} \mathrm{and} \mathrm{an} \mathrm{injection} \mathrm{energy}$ $\gamma=27$. We can observe that the amplitude of the longitudinal electric field in the SPS is much lower when compared to that of the PS, as a consequence of the increased beam rigidity.

\section{THE LONGITUDINAL INDIRECT SPACE CHARGE IMPEDANCE AND THE FORM FACTOR}

The indirect field given by Eq. (37) can also be seen as the field produced by the image currents moving inside the pipe wall. In this way, Eq. (37) is useful to evaluate the so-called longitudinal indirect space charge coupling impedance, which, for low energies, can represent a very important contribution to the total impedance of a machine [2]. Its value per unit of length can be written as [13]

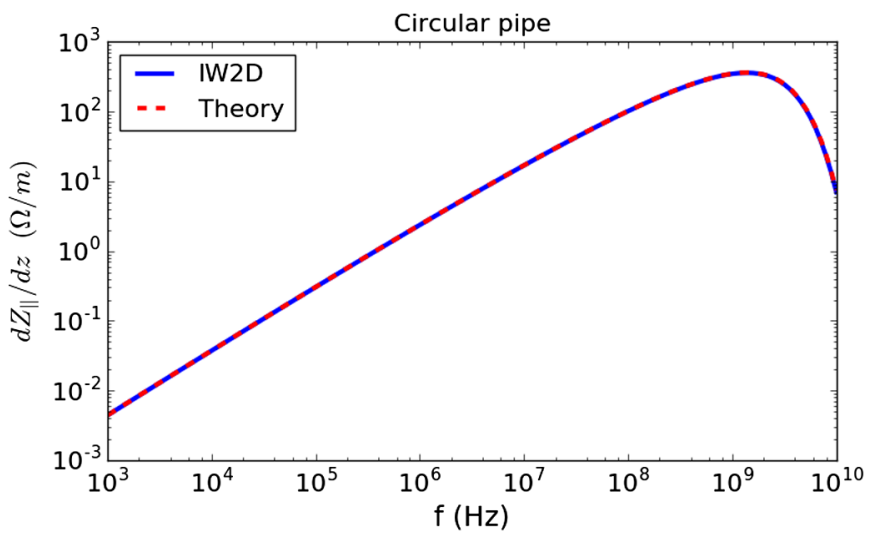

$$
\frac{d Z_{/ /}}{d z}=-\frac{E_{z}^{i}\left(\mu=0, \varphi=\frac{\pi}{2}\right)}{Q} .
$$

This impedance is purely imaginary and it can be used to evaluate the effect of indirect space charge for any elliptical geometry. In Fig. 6 we have compared Eq. (40), as a function of frequency, with the results given by the code IW2D [18], which has been developed to obtain the wall impedance (taking into account also the conductivity of the pipe wall) for circular and flat (parallel plates) geometries. For IW2D we have used a very low resistivity, while, for Eq. (38), we have considered two cases. In the first case, we assumed a less than $2 \%$ difference between the two axes of the ellipse (for approximating the round case, left side). In the second case, we assumed the ellipse major axis ten times larger than the minor one (for approximating the case of parallel plates, right side). In both cases we considered 120 summation terms, and we used $b=3.5 \mathrm{~cm}$ and $\beta=0.9$, which are of interest for the PS machine. As we can see from the two figures, the agreement between the two methods is very good.

Equation (40) has also been used to evaluate the ratio between the longitudinal impedance as a function of $q_{r}=(a-b) /(a+b)$, and the impedance of the corresponding circular pipe $\left(q_{r} \rightarrow 0\right)$. In Fig. 7 we show this form factor for different frequencies at the PS and the SPS injection energies. The black vertical lines correspond to the machines beam pipe geometries (for PS $q_{r}=0.35$, for SPS $\left.q_{r}=0.58\right)$. It is important to observe that the form factor depends on both the beam energy and the frequency. If, from Eq. (39), we take into account only the indirect space charge contribution, the longitudinal impedance at low frequency for a circular pipe can be written as [13]

$$
\frac{d Z_{/ /}}{d z}=-\frac{G}{Q}\left[\log \left(\frac{k_{0} r_{0}}{\beta \gamma}\right)+\gamma_{e}\right]
$$

with $\gamma_{e}$ the Eulers constant.

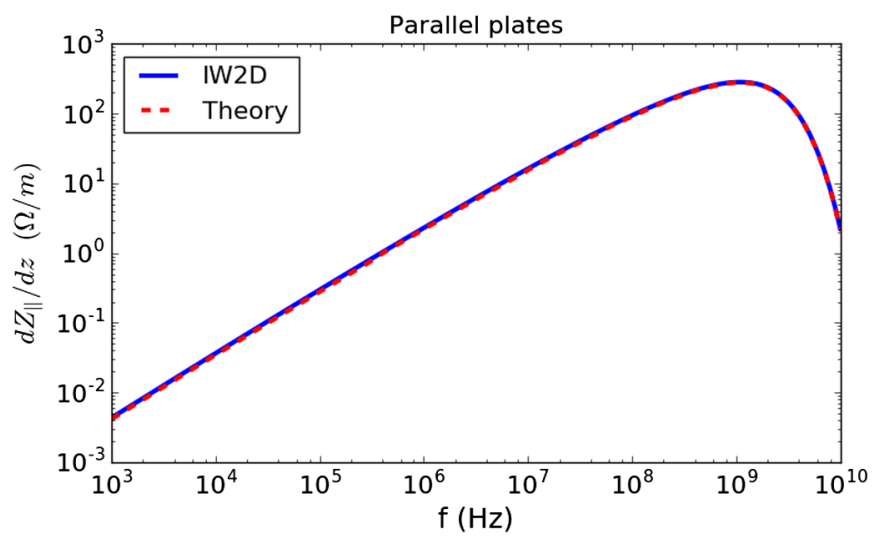

FIG. 6. Longitudinal indirect space charge per unit of length for $b=3.5 \mathrm{~cm}$ and $\beta=0.9$ as a function of frequency. Comparison between IW2D and Eq. (38) for circular pipe (left) and parallel plates (right). 

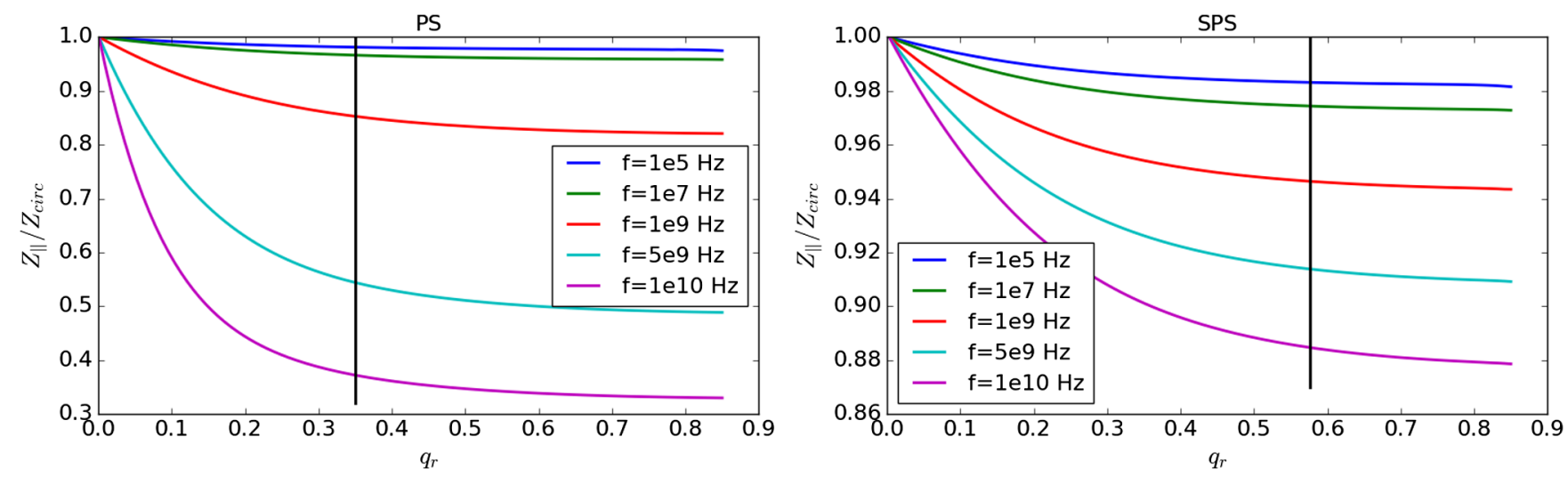

FIG. 7. Longitudinal form factor as a function of $q_{r}=(a-b) /(a+b)$ for the PS (left) and the SPS (right) injection energies at different frequencies. The vertical black lines correspond to the machines beam pipe geometries.

It is also possible to compare Eq. (40), in case of low frequency, with the results presented in [4]. In order to extend Eq. (41) to the elliptical case, we can substitute the pipe radius $r_{0}$ with the longitudinal equivalent radius defined in [4] as:

$$
r_{e q}=b \frac{4 \sqrt{q_{r}}}{\left(q_{r}-1\right) \vartheta_{2}^{2}\left(0, q_{r}\right)},
$$

with $\vartheta_{2}$ the Jacobi theta function. In Fig. 8, a comparison between the impedance given by Eq. (40) with that of Eq. (41) by using the equivalent radius (42) at low frequency $(100 \mathrm{kHz})$ for the PS injection energy at different $q_{r}$, is shown. As we can see, the agreement is very good.

\section{THE TRANSVERSE INDIRECT SPACE CHARGE QUADRUPOLAR IMPEDANCE}

From the indirect longitudinal electric field of Eq. (37), it is possible to obtain also the transverse indirect space charge quadrupolar coupling impedance in elliptic geometry. In our notation, dipolar and quadrupolar impedances

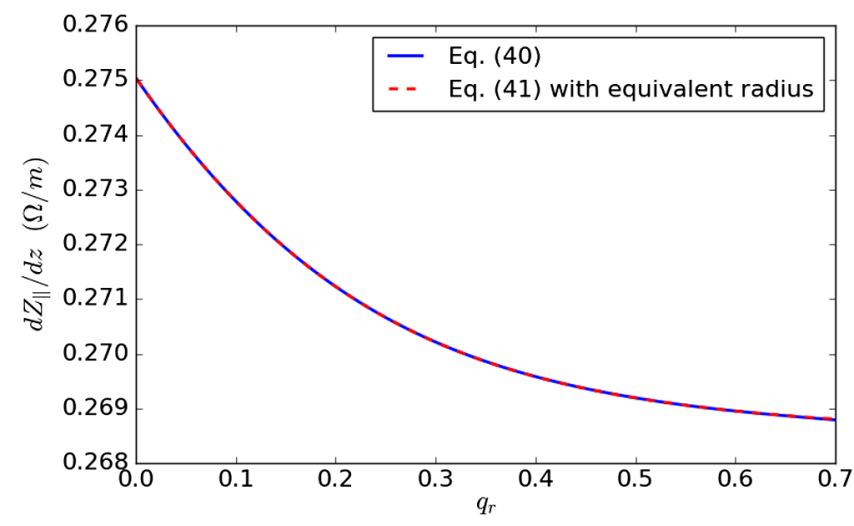

FIG. 8. Longitudinal indirect space charge impedance as a function of $q_{r}=(a-b) /(a+b)$ for the PS accelerator for a frequency of $100 \mathrm{kHz}$. Comparison between Eq. (40) and Eq. (41) by using the equivalent radius defined in Eq. (42). have the following definitions: the dipolar impedance is computed displacing the source particle and considering the wakefield effect on the test particle in the center. The quadrupolar impedance is computed displacing the test particle keeping the source particle in the center. In both cases, the dipolar or quadrupolar wakefield effect is linear with the source or test particle displacement [25]. Let us consider, as example, the vertical plane. The vertical impedance is defined as

$$
Z_{\perp, y}=\frac{j}{Q \Delta y} \int_{-\infty}^{\infty}\left(E_{y}^{i}+v B_{x}^{i}\right) d z
$$

where $v$ is the beam velocity.

The indirect fields in the above equation are generated by a point charge moving on the axis of the beam pipe, and they are evaluated in $(\varphi=\pi / 2, \mu=\Delta \mu)$, that is in $(x=0, y=\Delta y=F \sinh \Delta \mu)$ with $\Delta \mu \ll \mu_{0}$ [25]. From the Faraday law in the frequency domain, by considering the longitudinal dependence of the electric field with $z$, as stated at the beginning of Sec. II, we have

$$
B_{x}^{i}=\frac{j}{\omega}\left(\frac{\partial E_{z}^{i}}{\partial y}+j k_{z} E_{y}^{i}\right)
$$

that, substituted in Eq. (43), gives

$$
Z_{\perp, y}=-\frac{1}{Q \Delta y k_{z}} \int_{-\infty}^{\infty} \frac{\partial E_{z}^{i}}{\partial y} d z
$$

from which we obtain the transverse indirect space charge quadrupolar impedance per unit of length

$$
\frac{d Z_{\perp, y}}{d z}=-\frac{1}{Q \Delta y k_{z}} \frac{\partial E_{z}^{i}}{\partial y} .
$$

An analogous expression can be found also for horizontal plane. Since we are using the elliptic coordinates, we have 


$$
\frac{\partial E_{z}^{i}}{\partial y}=\frac{\partial E_{z}^{i}}{\partial \mu} \frac{\partial \mu}{\partial y}+\frac{\partial E_{z}^{i}}{\partial \varphi} \frac{\partial \varphi}{\partial y}
$$

The derivatives have to be done with constant $x$, so that, from Eq. (2), we have

$$
\begin{aligned}
\frac{\partial \mu}{\partial y} & =\frac{\cosh \mu \sin \varphi}{F\left(\sinh ^{2} \mu+\sin ^{2} \varphi\right)} \\
\frac{\partial \varphi}{\partial y} & =\frac{\sinh \mu \cos \varphi}{F\left(\sinh ^{2} \mu+\sin ^{2} \varphi\right)} .
\end{aligned}
$$

Since in $\varphi=\pi / 2$ the second of the above equations is zero, we remain only with the derivative in $\mu$. By using for the field Eq. (37), we finally obtain

$$
\begin{aligned}
\frac{d Z_{\perp, y}}{d z}= & \frac{2 \pi G}{Q k_{z}} \frac{1}{F^{2} \sinh \Delta \mu \cosh \Delta \mu} \\
& \cdot\left[\sum_{l=0}^{\infty} \frac{A_{0}^{(2 l)}}{p_{2 l}^{\prime}} c e_{2 l}(0, q) \frac{F e k_{2 l}\left(\mu_{0},-q\right)}{C e_{2 l}\left(\mu_{0},-q\right)}\right. \\
& \left.\times\left.\frac{d C e_{2 l}(\mu,-q)}{d \mu}\right|_{\mu=\Delta \mu}\right] .
\end{aligned}
$$

For the horizontal plane the field has to be evaluated in $\left(\varphi=\frac{\pi}{2}-\Delta \varphi, \mu=0\right)$ with $\Delta \varphi \ll 1$, and, by following the same procedure, we obtain

$$
\begin{aligned}
\frac{d Z_{\perp, x}}{d z}= & \frac{2 \pi G}{Q k_{z}} \frac{1}{F^{2} \sin \Delta \varphi \cos \Delta \varphi} \\
& \cdot\left[\sum_{l=0}^{\infty} \frac{A_{0}^{(2 l)}}{p_{2 l}^{\prime}} C e_{2 l}(0,-q) \frac{F e k_{2 l}\left(\mu_{0},-q\right)}{C e_{2 l}\left(\mu_{0},-q\right)}\right. \\
& \left.\times\left.\frac{d c e_{2 l}\left(\frac{\pi}{2}-\varphi, q\right)}{d \varphi}\right|_{\varphi=\frac{\pi}{2}-\Delta \varphi}\right] .
\end{aligned}
$$

As in the longitudinal case, the transverse quadrupolar impedance is purely imaginary. In Fig. 9 we have compared Eq. (50), as a function of frequency, with the results given by IW2D under the same assumptions as in the longitudinal plane of Fig. 6. We have also represented the impedance at different values of $q_{r}$. When $q_{r}>0.8$, we retrieve the flat chamber case. We also observe that the PS quadrupolar impedance due to space charge, for which $q_{r}=0.35$, can be much higher and not negligible with respect to the circular case. Also in this case, we find that the agreement between our method and IW2D is very good. It is important to highlight that at high frequency, the quadrupolar impedance of a circular pipe is different from zero. Only when $\beta \rightarrow 1$ this contribution disappears. In the same figure, we have also represented the quadrupolar transverse impedance obtained by using the Laslett coefficients for an elliptic pipe. The expression, valid only at low frequencies, gives a constant imaginary impedance that can be written as [26]

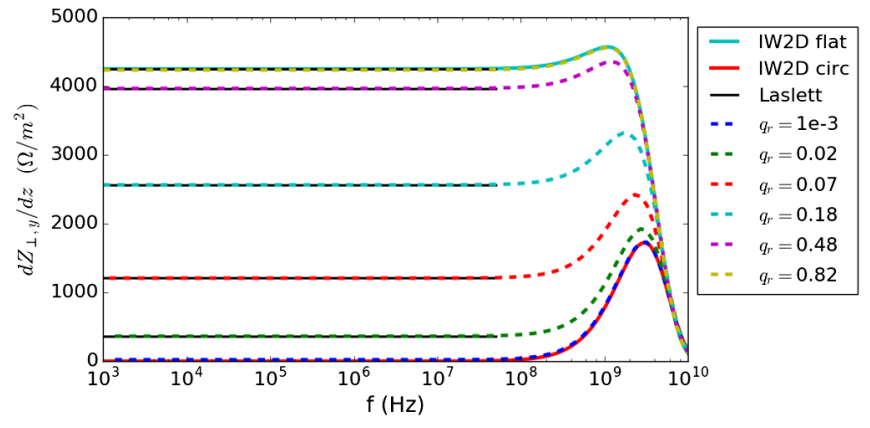

FIG. 9. Transverse indirect space charge per unit of length for $b=0.35 \mathrm{~cm}$ and $\beta=0.9$ as a function of frequency for different $q_{r}=(a-b) /(a+b)$ compared with the circular and flat beam pipe given by IW2D, and with theory which uses the Laslett coefficients at low frequency.

$$
\frac{d Z_{\perp, y}}{d z}=\frac{j Z_{0}}{\pi \gamma^{2} \beta b^{2}} \epsilon_{1, \gamma}
$$

where

$$
\epsilon_{1, \gamma}=\frac{b^{2}}{12 F^{2}}\left[\left(1+k^{\prime 2}\right)\left(\frac{2 K(k)}{\pi}\right)^{2}-2\right]
$$

with $K(k)$ the complete elliptic integral of the first kind, $k=\sqrt{1-k^{\prime 2}}$, and $k^{\prime}$ the complementary modulus given by

$$
k^{\prime}=\left(\frac{1+2 \sum_{s=1}^{\infty}(-1)^{s} q_{r}^{s^{2}}}{1+2 \sum_{s=1}^{\infty} q_{r}^{s^{2}}}\right)^{2} .
$$

Equation (53) is not suited to evaluate the impedance when the pipe geometry tends to be flat. Indeed, when $q_{r} \rightarrow 1, k \rightarrow 0$ and then $k \simeq 1$. Both $K(k)$ and $F$ tend to infinity and the evaluation of the equation needs particular care. This happens already when $q_{r} \geq 0.8$. However, the agreement with Eq. (50) at low frequency is very good.

\section{CONCLUSIONS}

In this paper, we have obtained a novel formula that describes the radiation process of a particle beam traveling along the longitudinal axis of a beam vacuum chamber of elliptical cross section. By using a convenient expansion of the Mathieu functions, we first obtained the longitudinal Green function in free space with elliptical coordinates, and then derived the indirect field due to the boundary elliptic geometry. The combination of the two fields, representing the total field, is expressed in terms of product series of periodic ordinary Mathieu functions $c e_{2 n}$ and radial modified Mathieu functions $\mathrm{Fe}_{2 n}$ and $\mathrm{Ce}_{2 n}$, and the final expression allows for an immediate computation of the longitudinal electromagnetic field inside the vacuum chamber when traversed by a particle beam. The equation, which can be evaluated for any elliptical geometry, for a test 
particle at any transverse position, and for any velocity of the charge, is written in closed form, thus allowing to be differentiated or integrated for further studies of electromagnetic interaction of a particle beam in an elliptic geometry. Theoretical results are in excellent agreement with those obtained with numerical simulations performed with CST Particle Studio in a variety of cases. This formula has been applied successfully to different realistic geometries of vacuum chambers installed in the CERN accelerator complex. By using the field in terms of Mathieu functions, we have also obtained the indirect space charge longitudinal and transverse quadrupolar coupling impedances for any elliptic geometry and frequency, and we have compared them with other existing analytical models, valid only at low frequency, and with the code IW2D for a circular pipe and parallel plates. The expression of the Green's function is useful since, from the knowledge of the fields in elliptical coordinates, the theory can be further developed to give, for example, the impedance due to the finite resistivity of an elliptic beam pipe wall, or to serve as a basis for an analytical model for the computation of the electric field generated by a point charge passing through a step transition between to confocal elliptical waveguides. The above problems are under investigation.

\section{ACKNOWLEDGMENTS}

We acknowledge Prof. Howard Wilson for the support given with the computation of Mathieu functions. We also acknowledge many helpful discussions with Dr. Elias Metral at CERN. This work has been supported in the framework of the CERN PS-LIU project. Work supported by the Director, Office of Science, of the U.S. Department of Energy under Contract No. DE-AC02-05CH11231.

\section{APPENDIX A: EIGENMODES EXPANSION IN ELLIPTIC WAVEGUIDE}

If we consider the eigenmodes in the elliptic waveguide, they satisfy the modal wave equation

$$
\nabla_{t}^{2} E_{z}^{r, s}+k_{r, s}^{2} E_{z}^{r, s}=0
$$

with $k_{r, s}^{2}$ the corresponding eigenvalues. These eigenmodes, which satisfy the homogeneous boundary conditions, allow to write the Green's function as

$$
E_{z}^{0}=\sum_{r, s=0}^{\infty} C_{r, s} E_{z}^{r, s}
$$

with $C_{r, s}$ the unknown coefficients. By introducing this equation in Eq. (19), and by using the orthonormal properties of the eigenmodes, it is possible to obtain, for the coefficients $C_{r, s}$, the expression

$$
C_{r, s}=-\frac{G}{\left(k_{t}^{2}-k_{p, t}^{2}\right)} E_{z}^{p, t}(0)
$$

It is important to observe that, since $k_{t}^{2}$ is negative, we will never obtain the resonance condition. Finally, the Green's function can be expressed as:

$$
E_{z}^{0}=-\sum_{r, s=0}^{\infty} \frac{G}{\left(k_{t}^{2}-k_{r, s}^{2}\right)} E_{z}^{r, s}(0) E_{z}^{r, s}
$$

However, in the problem discussed in this paper, this expansion would result more cumbersome because of the additional complexity of finding the eigenvalues of the Mathieu functions. Nevertheless, it is important to underline that this type of expansion can be extremely useful when it is necessary to use the orthogonality properties in order to solve some particular problems, as, for example, that of a step transition [27].

\section{APPENDIX B: GREEN FUNCTION IN ELLIPTIC COORDINATES IN THE LIMIT $\boldsymbol{F} \rightarrow \mathbf{0}$}

If we consider the elliptic pipe with $b \rightarrow a=r_{0}$, that is $F \rightarrow 0$, from Eq. (31) we also have that $q \rightarrow 0$. The coefficients $A_{0}^{(2 l)}$, when $q=0$, are all zero [8] except $A_{0}^{(0)}=1 / \sqrt{2}$, so that in Eq. (38) the summation over $l$ disappears, and the field becomes

$$
\begin{aligned}
E_{z}^{i}= & 2 \pi G \frac{A_{0}^{(0)}}{p_{0}^{\prime}} c e_{0}\left(\frac{\pi}{2}-\varphi, 0\right) \\
& \times\left(F e k_{0}(\mu, 0)-\frac{F e k_{0}\left(\mu_{0}, 0\right)}{C e_{0}\left(\mu_{0}, 0\right)} C e_{0}(\mu, 0)\right) .
\end{aligned}
$$

The ordinary Mathieu function of first kind $c e_{0}(\varphi, 0)$ has to be equal to $A_{0}^{(0)}$. Moreover, by using Eq. (15), we can write

$$
F e k_{0}(\mu, 0)=\frac{p_{0}^{\prime}}{\pi A_{0}^{(0)}} \sum_{r=0}^{\infty} A_{2 r}^{(0)} I_{r}\left(\nu_{1}\right) K_{r}\left(\nu_{2}\right) .
$$

Since also $A_{2 r}^{(0)}$ are all zero except the one with $r=0$, the summation over $r$ disappears, and we obtain

$$
F e k_{0}(\mu, 0)=\frac{p_{0}^{\prime}}{\pi A_{0}^{(0)}} I_{0}\left(\nu_{1}\right) K_{0}\left(\nu_{2}\right) .
$$

Being

$$
\nu_{1}=\sqrt{q} e^{-\mu} \rightarrow 0, \quad \nu_{2}=\sqrt{q} e^{\mu} \rightarrow \frac{k_{0} r}{\beta \gamma},
$$

we obtain 


$$
F e k_{0}(\mu, 0)=\frac{p_{0}^{\prime}}{\pi A_{0}^{(0)}} K_{0}\left(\frac{k_{0} r}{\beta \gamma}\right)
$$

Analogously, we can write

$$
F e k_{0}\left(\mu_{0}, 0\right)=\frac{p_{0}^{\prime}}{\pi A_{0}^{(0)}} K_{0}\left(\frac{k_{0} r_{0}}{\beta \gamma}\right) .
$$

Finally, we can express the radial modified Mathieu functions of the first kind in terms of product series of Bessel functions [8], as

$$
\begin{aligned}
C e_{0}(\mu, 0) & =\frac{p_{0}^{\prime}}{A_{0}^{(0)}} \sum_{r=0}^{\infty}(-1)^{r} A_{2 r}^{(0)} I_{r}\left(\nu_{1}\right) I_{r}\left(\nu_{2}\right) \\
& =\frac{p_{0}^{\prime}}{A_{0}^{(0)}} A_{0}^{(0)} I_{0}\left(\nu_{1}\right) I_{0}\left(\nu_{2}\right) \\
& =p_{0}^{\prime} I_{0}\left(\frac{k_{0} r_{0}}{\beta \gamma}\right)
\end{aligned}
$$

and

$$
C e_{0}(\mu, 0)=p_{0}^{\prime} I_{0}\left(\frac{k_{0} r}{\beta \gamma}\right) .
$$

If we substitute Eqs. (B5)-(B8) in Eq. (38), we obtain the longitudinal electric field generated by a point charge travelling on the axis of a perfectly conducting circular beam pipe of radius $r_{0}$, as given by Eq. (39).

[1] L. Palumbo, V. G. Vaccaro, and M. Zobov, Report No. LNF-94-041-P, 1994.

[2] M. Migliorati, H. Damerau, S. Gilardoni, S. Hancock, L. Palumbo, and S. Persichelli, Beam-wall interaction in the CERN Proton Synchrotron for the LHC upgrade, Phys. Rev. ST Accel. Beams 16, 031001 (2013).

[3] S. Persichelli, M. Migliorati, N. Biancacci, S. Gilardoni, E. Metral, and B. Salvant, Transverse beam coupling impedance of the CERN Proton Synchrotron, Phys. Rev. Accel. Beams 19, 041001 (2016).

[4] L. Palumbo, V. G. Vaccaro, and G. Wustefeld, Coupling impedance in a circular particle accelerator, a particular case: Circular beam, elliptic chamber, IEEE Trans. Nucl. Sci. 31, 1011 (1984).

[5] A. Lutman, R. Vescovo, and P. Craievich, Electromagnetic field and short-range wake function in a beam pipe of elliptical cross section, Phys. Rev. ST Accel. Beams 11, 074401 (2008).
[6] L. Palumbo and V. G. Vaccaro, The coupling impedance between a circular beam and a lossy elliptic vacuum chamber in particle accelerators, Nuovo Cimento Soc. Ital. Fis. 89A, 3 (1985).

[7] E. Mathieu, Memoire sur le mouvement vibratoire dune membrane de forme elliptique, J. Math. Pures Appl. 13, 137203 (1868).

[8] N.W. McLachlan, Theory and Application of Mathieu Functions (Clarendon Press, Oxford, 1951).

[9] J. C. Gutirrez-Vega, R. M. Rodrguez-Dagnino, M. A. Meneses-Nava, and S. Chvez-Cerda, Mathieu functions, a visual approach, Am. J. Phys. 71, 233 (2003).

[10] H. Wilson and R. Scharstein, Computing elliptic membrane high frequencies by Mathieu and Galerkin methods, J. Eng. Math. 57, 41 (2007).

[11] S. J. Zhang and Y. C. Shen, Eigenmode sequence for an elliptical waveguide with arbitrary ellipticity, IEEE Trans. Microwave Theory Tech. 43, 1 (1995).

[12] L. Chu, Electromagnetic waves in elliptic hollow pipes of metal, J. Appl. Phys. 9, 583 (1938).

[13] R. L. Gluckstern, CERN Report No. 2000-011, 2000.

[14] M. Abramowitz and I. Stegun, Handbook of Mathematical Functions with Formulas, Graphs, and Mathematical Tables (Dover, New York, 1964).

[15] J. C. Gutirrez-Vega, R. M. Rodrguez-Dagnino, and S. Chvez-Cerda, Free oscillations in an elliptic membrane, Rev. Mex. Fis. 45, 613 (1999).

[16] D. Davino, G. Dome, G. Miano, G. Panariello, V. G. Vaccaro, and L. Verolino, Longitudinal coupling impedance of an abrupt junction in a vacuum chamber, Nuovo Cimento Soc. Ital. Fis. 112A, 1401 (1999).

[17] A. Erdelyi, W. Magnus, F. Oberhettinger, and F. G. Tricomi, Higher Transcendental Functions (McGraw-Hill, New York, 1953), Vol. 2.

[18] N. Mounet, Ph.D. thesis, cole Polytechnique Fdrale de Lausanne, Lausanne (CH), 2012, p. 135.

[19] S. Persichelli, Ph.D. thesis, University of Rome La Sapienza, Rome (IT), 2015, p. 102.

[20] L. V. Kantorovich and V. I. Krylov, Approximate Methods of Higher Analysis (Interscience, New York, 1958).

[21] S. Kurennoy, Coupling impedances of small discontinuities: Dependence on beam velocity, Phys. Rev. ST Accel. Beams 9, 054201 (2006).

[22] A. Piwinski, Report No. DESY-94-068, 1994.

[23] https://www.cst.com.

[24] M. Benedikt, P. Collier, V. Mertens, J. Poole, and K. Schindl, LHC Design Report No. CERN-2004-003, 2004.

[25] S. Heifets, A. Wagner, and B. Zotter, Report No. SLAC/ AP110, 1998.

[26] K. Y. Ng, Physics of Intensity Dependent Beam Instabilities (World Scientific, Singapore, 2006).

[27] G. Dome, E. Gianfelice, L. Palumbo, V. G. Vaccaro, and L. Verolino, Il Nuovo Cimento A 104, 1241 (1991). 